\title{
Abnormal Bedside Ultrasound Findings in a Complex Patient With Kawasaki Disease
}

\author{
Dipal Shah ${ }^{1}$, Helene Koumans ${ }^{2}$, Kimberly Johnson ${ }^{1}$, Leoh N. Leon ${ }^{3,1}$, Latha Ganti 4, 5, 1, 6 \\ 1. Emergency Medicine, Ocala Regional Medical Center, Ocala, USA 2. Emergency Medicine, Brown University, \\ Providence, USA 3. Emergency Medicine, Osceola Regional Medical Center, Orlando, USA 4. Emergency Medicine, \\ Envision Physician Services, Plantation, USA 5. Emergency Medicine, University of Central Florida College of Medicine, \\ Orlando, USA 6. Emergency Medicine, HCA Healthcare Graduate Medical Education Consortium Emergency Medicine \\ Residency Program of Greater Orlando, Orlando, USA
}

Corresponding author: Latha Ganti, latha.ganti@ucf.edu

\begin{abstract}
We herein report a case of an 18-year-old female with Kawasaki disease who presented to the emergency department with epigastric abdominal pain and was subsequently found to have free fluid present in her abdomen visualized on bedside Focused Assessment with Sonography for Trauma (FAST) exam. Kawasaki disease is an acute vasculitis syndrome that primarily affects children and can have serious complications such as coronary artery aneurysms. The use of ultrasound in emergency departments is rapidly increasing, with the FAST being one of the most commonly performed bedside ultrasound examinations. FAST exams are most commonly performed in trauma patients as well as being part of the Advanced Trauma Life Support (ATLS) protocol. However, this case demonstrated that the FAST exam can also have application in other clinical scenarios and patient presentations where there is clinical suspicion of free intra-abdominal fluid.
\end{abstract}

Review began 07/28/2021 Review ended 07/31/2021 Published 08/11/2021

\section{(๑) Copyright 2021}

Shah et al. This is an open access article distributed under the terms of the Creative Commons Attribution License CC-BY 4.0., which permits unrestricted use, distribution, and reproduction in any medium, provided the original author and source are credited.
Categories: Cardiology, Emergency Medicine, Radiology

Keywords: kawasaki disease, focused assessment with sonography for trauma, abdominal pain, pocus (point of care ultrasound, epigastric pain

\section{Introduction}

Kawasaki disease (KD), also termed mucocutaneous lymph node syndrome, is an acute vasculitis syndrome primarily affecting small to medium-sized arteries of children under five years old. Although the etiology is unknown, patients with KD frequently present with fever, swelling of the hands and feet, rash, swollen glands in the neck, and irritation of the mouth, lips, throat, and eyes.

KD causes inflammation in artery walls, and serious complications include coronary artery dilations and aneurysms (CAA). KD is a leading cause of acquired heart disease in the United States and the leading cause of pediatric ischemic heart disease, though a standard treatment of intravenous immunoglobulin and aspirin has been developed to stifle the development of coronary artery abnormalities [1].

Mild dilation or ectasia of the coronary arteries occurs in up to approximately $40 \%$ of patients, and coronary artery aneurysms occur in $15-25 \%$ of untreated patients. The standard treatment of intravenous immunoglobulin and aspirin, an anti-platelet medication, significantly decrease the development of coronary artery complications. Additionally, coronary artery bypass grafting (CABG) has proven safe and effective for KD-associated coronary heart disease.

The subsequent report describes a case of a patient presenting with epigastric pain and significant free fluid upon conducting a Focused Assessment with Sonography for Trauma (FAST) scan, which assesses the pericardium and three spaces within the peritoneal cavity for fluid, and has proven to be quite sensitive. The symptoms of the case were of particular note due to the history of $\mathrm{KD}$, CAA, and CABG.

\section{Case Presentation}

An 18-year-old female with history of KD, coronary artery aneurysms, and coronary artery bypass grafting $(C A B G)$ presents to the emergency department by EMS reporting abdominal pain starting earlier this morning. The patient describes the abdominal pain as epigastric radiating to her right shoulder and worsened with deep inspiration. The pain has been worsening throughout the day. She also endorses nausea. She denies vaginal bleeding and reports that her last menstrual period was approximately one month ago. She endorses a history of bulimia, and reports forceful vomiting episodes every day for the past year. Otherwise, the patient denies fever, cough, recent illness, trauma or injury. She notably has a history of KD as a child resulting in coronary artery aneurysms and requiring CABG surgery at age 12 . She is currently taking warfarin and aspirin.

Physical exam is remarkable for blood pressure of $99 / 69 \mathrm{mmHg}$ and heart rate of 98 beats per minute, 


\section{Cureus}

remaining vital signs within normal limits. On exam, the patient is in no acute distress. The exam is significant for diffuse abdominal tenderness with voluntary guarding.

Due to significant abdominal tenderness on initial physical exam, bedside FAST scan was performed, which was found to be positive, demonstrating a significant amount of free fluid in the right upper quadrant, left upper quadrant, and suprapubic views (Figures 1,2).
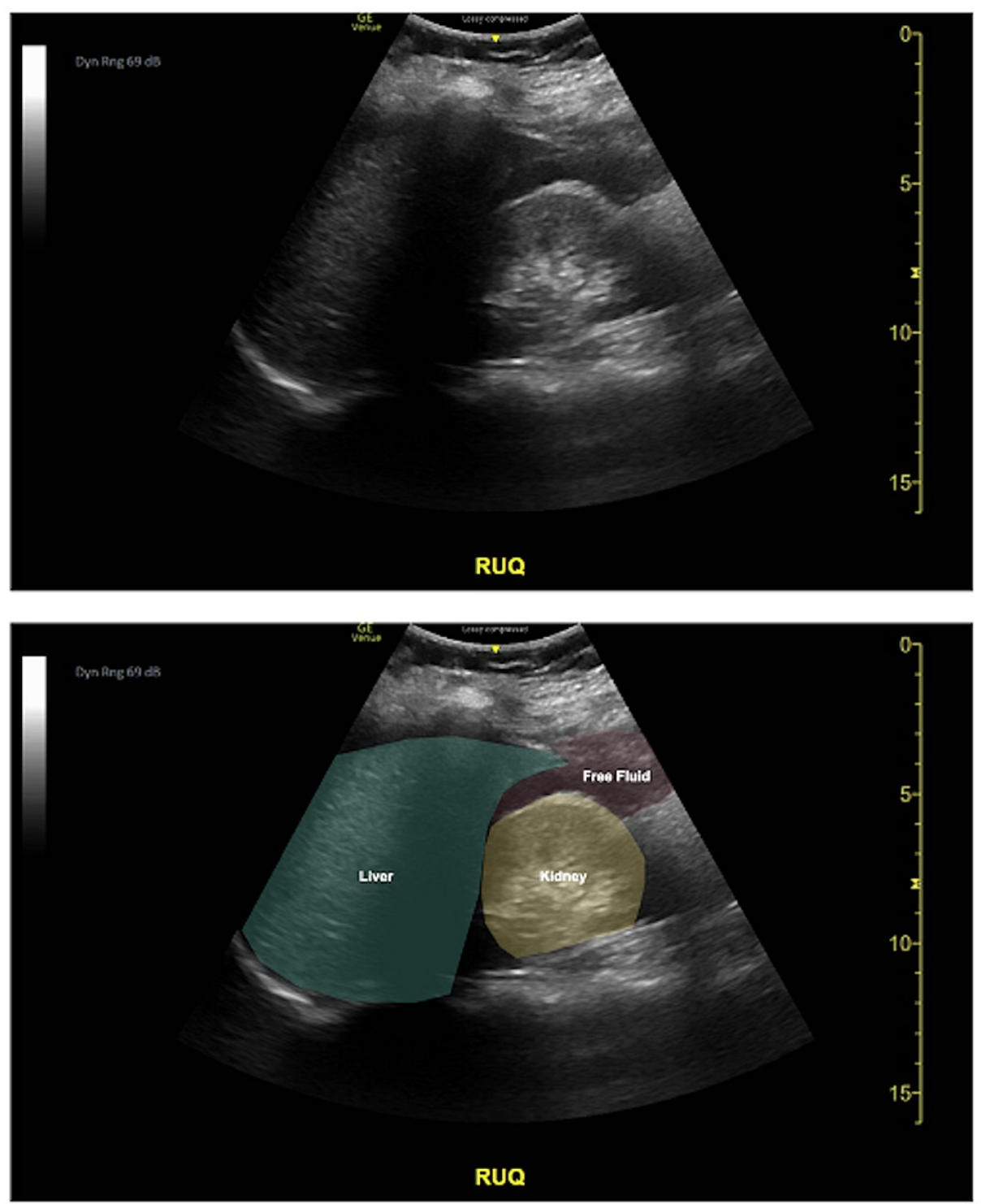

FIGURE 1: Right upper quadrant view seen on bedside ultrasonography. Transducer was placed at the mix-axillary line at approximately the 10th rib with the probe indicator towards the patient's head visualizing the diaphragm, liver, kidney and a potential space known as "Morrison's Pouch." This image demonstrates fluid, visualized as an anechoic (black) area inside of Morrison's Pouch. The lower image is a colorcoded version of the original image for visual interpretation. 


\section{Cureus}
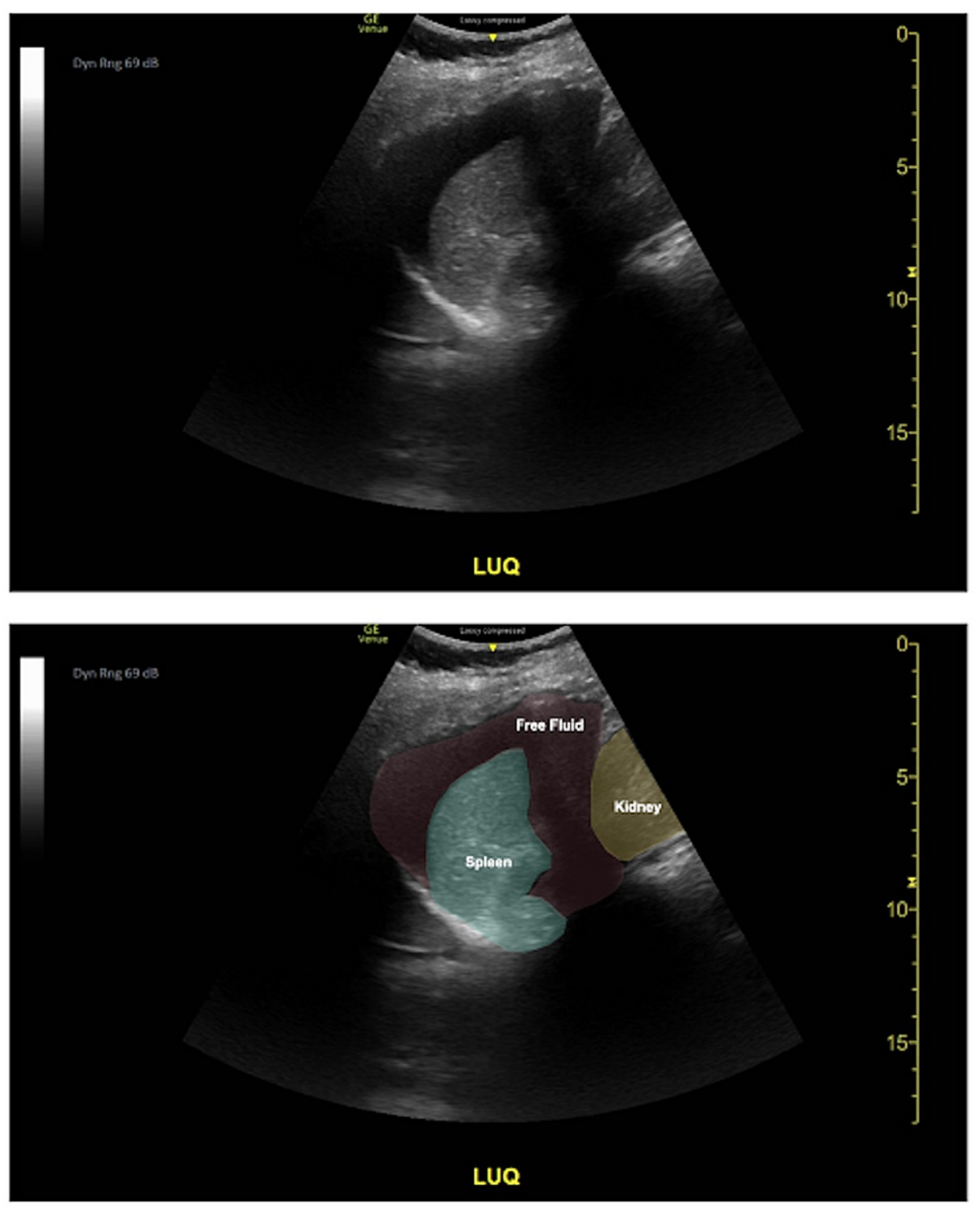

FIGURE 2: Left upper quadrant view seen on bedside ultrasonography. To obtain this view, the transducer is placed on the posterior axillary line at approximately the 8th rib as the spleen is more posterior and superior in relation to the liver. This image is similar to that of the RUQ and demonstrates the diaphragm, spleen, kidney. In this image we appreciate an anechoic (black) area between the spleen and kidney as well as superiorly to the spleen. The lower image is a color-coded version of the original image for visual interpretation.

Other workup was remarkable for anemia with a hemoglobin of $8.0 \mathrm{~g} / \mathrm{dl}$ and negative pregnancy test. Surgery was consulted and computed tomography angiography (CTA) of the chest, abdomen, pelvis were performed emergently due to concern for internal bleeding. CTA imaging demonstrated no acute findings of the aorta, and coronary artery aneurysms at the right coronary artery (RCA) and left anterior descending artery (LAD). Non-contrast CT of the abdomen and pelvis demonstrated free fluid (Figure 3). 


\section{Cureus}

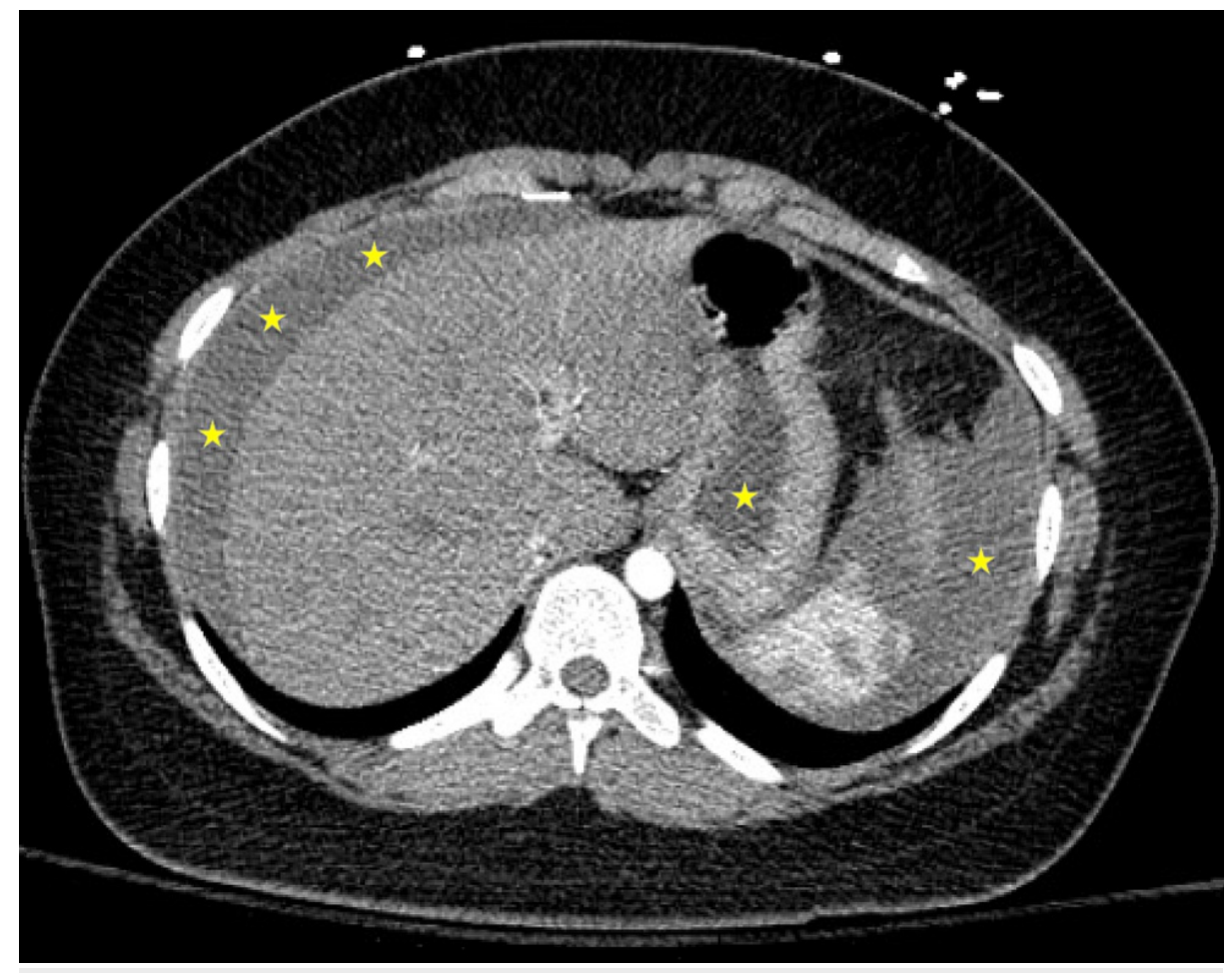

FIGURE 3: Non-contrast computed tomography (CT) scan demonstrating free fluid (asterisks).

Prothrombin complex concentrate (PCC) and tranexamic acid (TXA) were given due to concern for bleeding on anticoagulation. Due to ongoing concern for internal bleeding along with the patient's risk factors, the patient was admitted to the surgical intensive care unit (ICU) for further management.

In the ICU, the patient was transfused 2 units of packed red blood cells. Serial hemoglobin checks and abdominal exams were performed with a plan for diagnostic laparoscopy if hemoglobin decreased. However, the patient's hemoglobin remained stable, abdominal pain improved, and the patient was eventually discharged to home the following day with close follow-up.

\section{Discussion}

The case presented with abdominal pain and a history of pediatric KD resulting in coronary artery aneurysms (Figure 4). The patient was already taking warfarin and aspirin. Aspirin is a standard treatment for $\mathrm{KD}[2-4]$, and there is ongoing inquiry into the safety and efficacy of warfarin as an accompanying treatment $[5,6]$. 


\section{Cureus}

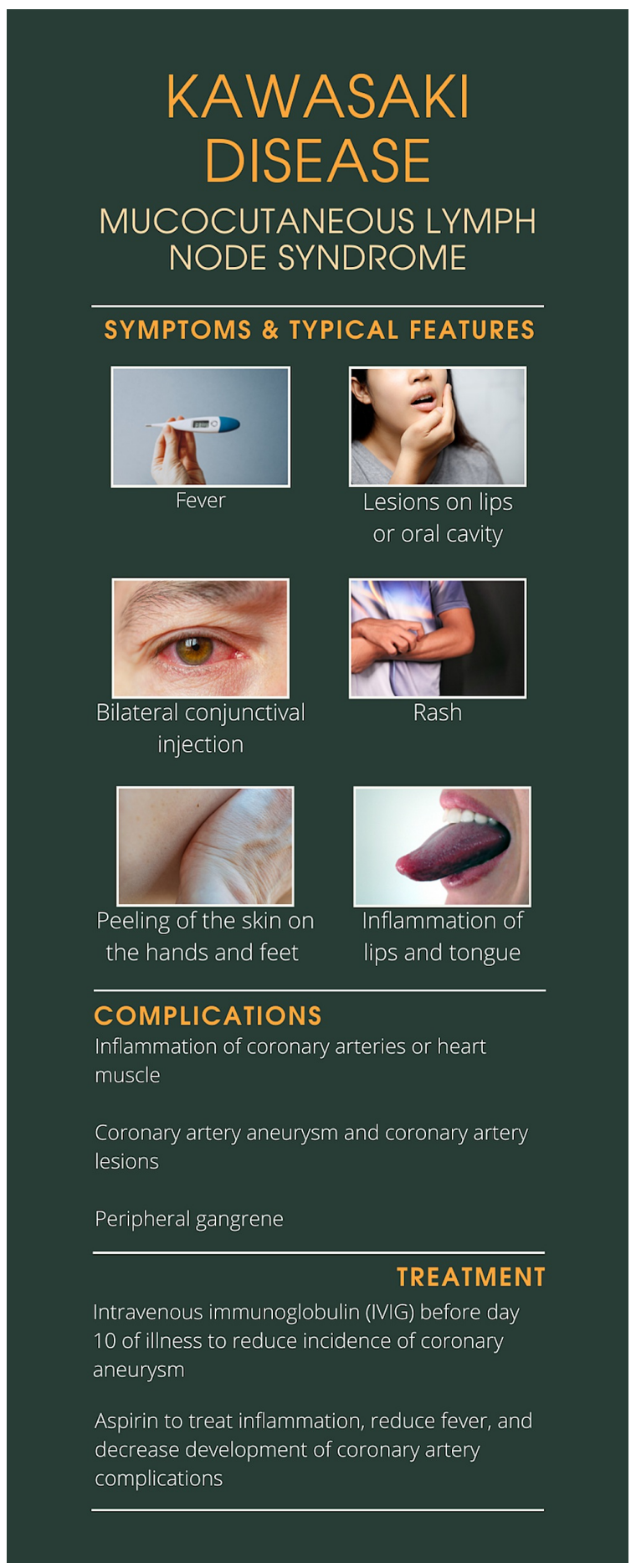

FIGURE 4: Infographic depicting typical features of Kawasaki disease.

Designed by Helene Koumans on Canva.

In addition to the patient's initial physical exam, a bedside FAST scan was emergently performed upon the patient's arrival via ambulance, after which a CTA was performed out of concern for internal hemorrhage, which may occur in KD patients secondary to a ruptured aneurysm. The FAST scan indicated a significant amount of free fluid, and prothrombin complex concentrate (PCC) and tranexamic acid (TXA) were administered due to concern for internal bleeding in a patient on anticoagulants. At present, there has not been considerable investigation of the use of FAST scans in cases of KD or those with history of KD. Further research has been conducted in adjacent fields as a result of the KD-like symptoms of the rare multisystem inflammatory syndrome in children reported as a post-infectious complication of severe acute respiratory syndrome coronavirus 2 (SARS-CoV-2), now termed pediatric inflammatory multisystem syndrome temporally associated with SARS-CoV-2 (PIMS-TS) [7]. 
FAST scans have demonstrated sensitivities between $85 \%$ and $96 \%$ and specificities above $98 \%$. Additionally, FAST scans can be conducted in fewer than five minutes and decrease time to surgical intervention [8]. Further study should be done of the benefits and risks of FAST scans in cases presenting abdominal pain and with a history of KD and coronary artery aneurysm. In this case, the FAST scan was critical in the identification of significant free fluid, which allowed for expedient follow-up laboratory work and treatment. Additional study of FAST scans with similar case presentations should be conducted to determine follow-up interventions necessary.

\section{Conclusions}

Prompt identification of free fluid and possible internal hemorrhage is critical in timely administration of follow-up treatment in the emergency department. Untreated internal bleeding, which may be exhibited in $\mathrm{KD}$ patients or patients with a history of KD, may result in organ failure, seizure, coma, and death. The performance of a FAST scan allowed for expedient identification of free fluid, subsequently allowing for necessary laboratory work and treatment. Thus, particularly among patients with a history of KD and presenting with abdominal pain, further study should be conducted on the performance of FAST scans in the determination of necessary intervention.

\section{Additional Information \\ Disclosures}

Human subjects: Consent was obtained or waived by all participants in this study. HCA Centralized Algorithms for Research Rules on IRB Exemptions (CARRIE) issued approval 2021-518. HCA Centralized Algorithms for Research Rules on IRB Exemptions (CARRIE)/ IRB manager issued approval [2021-518]. Based on the information provided and attested as true, the research plan described does not require IRB oversight. This is because you are either a) not engaging in research with human subjects as defined by federal regulations; b) engaging in research with human subjects deemed excluded from IRB oversight per 45CFR46.102(l) OR c) engaging in research with sufficient human subject protections in the design to meet one or more IRB exemption criteria set forth in 45CFR46.104. Conflicts of interest: In compliance with the ICMJE uniform disclosure form, all authors declare the following: Payment/services info: All authors have declared that no financial support was received from any organization for the submitted work. Financial relationships: All authors have declared that they have no financial relationships at present or within the previous three years with any organizations that might have an interest in the submitted work. Other relationships: All authors have declared that there are no other relationships or activities that could appear to have influenced the submitted work.

\section{Acknowledgements}

This research was supported (in whole or in part) by HCA Healthcare and/or an HCA Healthcare affiliated entity. The views expressed in this publication represent those of the author(s) and do not necessarily represent the official views of HCA Healthcare or any of its affiliated entities.

\section{References}

1. Modesti AM, Plewa MC: Kawasaki Disease. StatPearls Publishing, Treasure Island; 2021.

2. Ishii M, Ebato T, Kato H: History and future of treatment for acute stage Kawasaki disease . Korean Circ J. 2020, 50:112-9. 10.4070/kcj.2019.0290

3. Wang J, Chen H, Shi H, et al.: Effect of different doses of aspirin on the prognosis of Kawasaki disease . Pediatr Rheumatol Online J. 2020, 18:48. 10.1186/s12969-020-00432-X

4. Zheng X, Yue P, Liu L, et al.: Efficacy between low and high dose aspirin for the initial treatment of Kawasaki disease: current evidence based on a meta-analysis. PLoS One. 2019, 14:e0217274. 10.1371/journal.pone.0217274

5. Baker AL, Vanderpluym C, Gauvreau KA, et al.: Safety and efficacy of warfarin therapy in Kawasaki disease . J Pediatr. 2017, 189:61-5. 10.1016/j.jpeds.2017.04.051

6. Levin M, Burns JC, Gordon JB: Warfarin plus aspirin or aspirin alone for patients with giant coronary artery aneurysms secondary to Kawasaki disease?. Cardiology. 2014, 129:174-7. 10.1159/000366052

7. Penner J, Abdel-Mannan O, Grant K, et al.: 6-month multidisciplinary follow-up and outcomes of patients with paediatric inflammatory multisystem syndrome (PIMS-TS) at a UK tertiary paediatric hospital: a retrospective cohort study. Lancet Child Adolesc Health. 2021, 5:473-82. 10.1016/S2352-4642(21)00138-3

8. Focused assessment with sonography for trauma . (2020). Accessed: July 25, 2021: https://www.statpearls.com/nursepractitioner/ce/activity/31414. 